\title{
MOTIVAÇÃO, SUCESSO E TRANSIÇÃO PARA O ENSINO SUPERIOR
}

\author{
Ângela Sá Azevedo ${ }^{1}$ \\ Luisa Faria ${ }^{2}$
}

Resumo: A transição do ensino secundário para o ensino superior apresenta-se ao jovem, simultaneamente, como um desafio e uma ameaça, considerando quer as restritas condições de acesso ao ensino superior, quer as diferentes exigências e descontinuidades existentes entre estes dois ciclos de ensino.

Surge, então, uma questão nuclear: Será que os alunos mais motivados (com um auto-conceito e uma auto-estima positivos, bem como atribuições internas, estáveis e controláveis para os seus resultados) são os que se adaptam melhor ao ensino superior e apresentam maior sucesso académico?

No sentido de responder a esta questão, foram, por nós, realizados vários estudos, dos quais vamos apresentar, neste trabalho, os resultados mais representativos, a saber: os correlacionais, com uma amostra de 649 alunos do $12 .^{\circ}$ ano (avaliação da relação entre motivação e sucesso académico), e os de teste-reteste, com uma amostra de 62 alunos do ensino superior, sendo de realçar que encontramos correlações positivas e significativas, especialmente entre o auto-conceito académico e o sucesso académico, tendo-se, ainda, constatado mudanças intra-individuais do auto-conceito, num sentido mais positivo.

Palavras-chave: transição, motivação, adaptação.

Motivation, Success, and Transition to University (Abstract): The transition from high school to university is simultaneously a challenge and a threat due either to the access constraints to higher education, and also to the gap between the two education cycles.

One should ask a central question: Will the highly motivated students (those with positive self-concept, positive self-esteem, and stable and controllable internal attributions to their performance) be more fitted to university? Will they achieve higher academic scores?

\footnotetext{
${ }^{1}$ Doutoranda em Psicologia na Faculdade de Psicologia e de Ciências da Educação da Universidade do Porto (FPCEUP). aazevedosts@mail.telepac.pt

2 Professora Associada com Agregação da FPCEUP. lfaria@fpce.up.pt Faculdade de Psicologia e de Ciências da Educação, Universidade do Porto, Rua Dr. Manuel Pereira da Silva, s/n - 4200-392 Porto.
} 
In order to answer these questions several studies were made using a sample of 649 high school students (for the evaluation of the relation between motivation and success) and 62 undergraduate students (for a test-retest study). The more relevant and significant findings are discussed in this paper, such as the significant correlations between academic self-concept and academic success and the positive intra-subject change in self-concept.

Keywords: transition, motivation, adaptation.

\section{Introdução}

A problemática da transição escolar tem vindo a ser desenvolvida na literatura, nas duas últimas décadas, de uma forma cada vez mais aprofundada e específica, realçando, sistematicamente, as dificuldades e os desafios que parecem estar associados à necessidade de lidar com condições novas $\mathrm{e}$ por vezes adversas. Neste quadro, a entrada para a universidade é para a maioria dos jovens o concretizar de um sonho, no entanto, poderá desencadear dificuldades de adaptação ao novo contexto e, até, insucesso académico: de facto, até as diferentes e constantes revisões dos planos de estudo do ensino secundário acabam por acrescentar discrepâncias e dificuldades na progressão entre o ensino secundário e o ensino superior.

Concretamente, no nosso país, o alargamento da escolaridade obrigatória até aos 15 anos, para além de possibilitar uma escolaridade mínima para os nossos jovens, provocou um incremento significativo do número de alunos que não conseguem atingir o final da escolaridade obrigatória com o $9 .^{\circ}$ ano completo, pautando-se pela mera frequência, sem aproveitamento ou com um aproveitamento baixo: e, nesta última circunstância, os alunos podem prosseguir estudos para o secundário, com uma preparação muito aquém da exigida por este nível de estudos, implicando, muitas vezes, dificuldades de adaptação, insucesso e consequente abandono escolar.

Ao mesmo tempo, a transição do $3 .^{\circ}$ ciclo para o secundário implica a tomada de decisões vocacionais pelos alunos, decisões estas nem sempre devidamente orientadas por profissionais de psicologia, uma vez que muitas das nossas escolas não contam com a colaboração de psicólogos. Assim sendo, as opções dos alunos poderão pautar-se pela procura de um percurso abrangente que permita o acesso a um leque alargado de profissões, pela fuga a determinadas disciplinas ou, ainda, pela expectativa de vir a frequentar um curso mais fácil e que implique menor empenho pessoal.

Deste modo, a transição do ensino secundário para o ensino superior surge como uma das mais importantes, não só porque os alunos, muitas vezes, se encontram marcados por um ensino secundário "livresco" e rígido, 
mas também porque irão enfrentar situações novas e difíceis, como condições de selecção e acesso desumanizadas (como é o caso da excessiva importância atribuída aos exames finais nacionais), numerus clausus obsoletos e insuficientes, bem como expectativas de emprego negativas e frustrantes, aspectos estes que podem pôr em causa o esforço despendido pelos alunos, durante inúmeros anos de escolaridade.

$\mathrm{Na}$ verdade, grande parte dos estudos salienta que o aluno em transição para o ensino superior pode encarar de forma negativa as diversas mudanças que a caracterizam, vindo a sofrer desadaptações que se podem traduzir em baixo investimento e consequente insucesso académico (Fisher, 1986, citado por Azevedo \& Faria, 2001).

Segundo Vaz Serra (1999), a transição escolar pode igualmente ser avaliada por alguns sujeitos como indutora de stress, de tensão, porque é vista como ameaçadora, ou seja, é antecipada pelo sujeito como desagradável; pode ainda ser descrita como desafiante, uma vez que exige, por parte dos estudantes, a adopção de estratégias de trabalho e organização pessoal diferentes daquelas que os jovens do secundário exercitam no seu dia-a-dia de estudantes. No entanto, a transição, independentemente de ser percepcionada como ameaçadora ou como desafiante, implica sempre a adopção por parte do indivíduo de estratégias para fazer face à mudança.

Uma análise atenta das tarefas desafiantes associadas à transição permite concluir que esta problemática ultrapassa a esfera pessoal do estudante, englobando aspectos contextuais, como a preparação dos professores do ensino superior para lidarem eficazmente com uma massa de alunos heterogénea e a preparação que o ensino secundário faculta aos alunos em transição, ou seja, o estudo da transição escolar do ensino secundário para o ensino superior deverá inserir-se numa perspectiva ecológica do desenvolvimento humano, partindo de um sujeito dinâmico que estabelece ligações bidireccionais com o seu contexto (Bronfenbrenner, 1979, 1981).

É, pois, neste cenário complexo que os jovens têm que saber despoletar e criar meios para enfrentar os desafios e as adversidades, pelo que o conhecimento das características motivacionais dos jovens, promotoras da adaptação ao ensino superior e do sucesso académico, emerge como fundamental para desenvolver estratégias de promoção do bem-estar psicossocial global de adolescentes e jovens adultos.

Assim, os dois grandes objectivos do presente artigo são a apresentação de resultados sobre a relação entre a motivação e o sucesso académico (em alunos em transição para o ensino superior, nomeadamente no que se refere ao auto-conceito, à auto-estima e às atribuições e dimensões causais), bem como realçar os aspectos da mudança intra-individual nas características motivacionais, em função da transição para o ensino superior, no âmbito de um estudo de teste-reteste com um ano de intervalo. 


\section{Características motivacionais e transição}

\section{Auto-conceito e auto-estima: definição e delimitação conceptual}

O auto-conceito e a auto-estima são constructos que têm suscitado grande interesse por parte dos investigadores, pelo que a literatura apresenta um conjunto alargado de definições, das quais salientaremos apenas as mais representativas.

Um dos autores mais referenciados nesta temática é Rosenberg, que definiu o auto-conceito como "the totality of individual's thoughts and feelings having reference to himself as an object" (1979, p. 7). Para este autor, é necessário distinguir três tipos de auto-conceito: o "extant self" (a forma como o sujeito se vê), o "desired self" (a forma como o sujeito gostaria de se ver) e o "presenting self" (a forma como o sujeito se apresenta aos outros).

Esta noção enfatiza o auto-conceito e a auto-estima como sendo indiscriminados, contrariamente ao que defendem a maior parte dos autores, isto é, que o auto-conceito e a auto-estima são constructos distintos, embora relacionados entre si.

Mais tarde, Vaz Serra (1988) construiu uma definição de auto-conceito integradora de perspectivas anteriores, definindo-o como "a percepção que o indivíduo tem de si próprio" (p. 127), como sendo "um ser físico, social e espiritual ou moral" (Gecas, 1982, cit. por Vaz Serra, 1986). Assim, assistimos, com este autor, à eleição de uma noção de auto-conceito que, apesar de sofrer influências oriundas do facto de o sujeito interagir, se vai mantendo relativamente constante, conferindo identidade aos seus actos (Vaz Serra, 1988; Vaz Serra, Firmino \& Ramalheira, 1988).

Tratando-se de um conceito complexo, Vaz Serra (1988) admitiu que, na formação do auto-conceito, podemos encontrar factores como as apreciações que os outros fazem sobre o nosso comportamento, o nosso desempenho em situações específicas, a comparação do nosso comportamento com o de outros significativos e o confronto entre o comportamento do sujeito e os valores do seu grupo de pertença.

Mais recentemente, Faria e Fontaine (1990), igualmente numa perspectiva integradora, definiram, em termos gerais, o auto-conceito como a "percepção que o sujeito tem de si próprio" e, em termos específicos, "como o conjunto de atitudes, sentimentos e conhecimento acerca das capacidades, competências, aparência e aceitabilidade social próprias" (p. 98). Segundo estas autoras, a noção de si próprio sofre influências das experiências que ocorrem nos diversos contextos, os quais, por sua vez, influenciam o comportamento e a acção do sujeito. 
Já do ponto de vista de Dias e Fontaine (2001, p. 256), “o auto-conceito compreende potenciais desejos, valores, objectivos, receios, ou seja, um conjunto de imagens possíveis que poderão variar conforme o contexto específico em que o indivíduo se encontra, sendo, na sua globalidade, tanto regulador do comportamento presente, como um conjunto de referenciais que permitem dar significado ao comportamento passado e motivar o comportamento futuro".

Assim, considerando que o auto-conceito representa as cognições do sujeito sobre si próprio, em diferentes contextos, compreende-se que se trata de um constructo multidimensional, dadas as implicações que as diferentes situações podem provocar a nível do mesmo (Peixoto \& Almeida, 1999).

Quanto à caracterização do auto-conceito, e numa tentativa de apresentar uma síntese das diversas características que têm vindo a ser apontadas na literatura, consideraremos os seus sete aspectos fundamentais, realçados por Shavelson, Hubner e Stanton (1976), de tal modo que o auto-conceito será: (i) organizado e estruturado, isto é, o sujeito retira das suas experiências sínteses que permitem estruturar e simplificar as mesmas, atribuindo-lhes um sentido; (ii) multifacetado ou multidimensional, ou seja, as diferentes experiências do sujeito organizam-se em facetas específicas; (iii) hierárquico, isto é, considera-se a existência de um auto-conceito com uma dimensão geral e com dimensões específicas, de acordo com as experiências individuais do sujeito. Assim, no topo da hierarquia, encontramos o auto-conceito geral, que se divide em académico e não académico. Por sua vez, o auto-conceito académico dissocia-se em áreas específicas relacionadas com a aprendizagem, como, por exemplo, a verbal e a matemática, enquanto o auto-conceito não académico se decompõe em social, físico e emocional, aspectos estes que se subdividem ainda noutros mais específicos; (iv) está$v e l$, sendo de salientar que o auto-conceito assume uma maior estabilidade no topo da hierarquia, dependendo cada vez mais do contexto, à medida que descemos para a base; (v) desenvolvimental, isto é, ao longo do seu desenvolvimento, o sujeito abandona as categorias mais gerais do auto-conceito e passa a utilizar categorias mais específicas, de acordo com as suas características; (vi) avaliativo, ou seja, o sujeito, para além de desenvolver descrições de si próprio (componente descritiva) nas diferentes situações, desenvolve também avaliações sobre si próprio nessas mesmas situações (componente avaliativa ou auto-estima), aspectos estes que são separáveis, em termos conceptuais, mas dificilmente o serão em termos empíricos; e, por fim, (vii) diferenciável, isto é, embora relacionado com outros constructos, não deixa de ser distinto dos mesmos, como, por exemplo, da realização escolar, como analisaremos no ponto seguinte.

Refira-se, ainda, que, contrariamente ao auto-conceito, que assume um carácter essencialmente cognitivo, a auto-estima refere-se a aspectos 
especificamente avaliativos e emocionais (Vaz Serra, 1988), pelo que se poderá pensar que se encontra associada à dimensão avaliativa do auto-conceito, tal como defenderam vários autores (Burns, 1982; Fleming \& Courtner, 1984; Gurney, 1986; Wells \& Marwell, 1976). Na mesma linha de pensamento, Wylie (1974) considerou que a auto-estima corresponderia a uma das três partes do auto-conceito, isto é, às avaliações acerca de aspectos restritos do $e u$, parte esta que complementaria o conceito geral de si mesmo e o "eu ideal".

Ou seja, pode afirmar-se que a auto-estima é encarada como um conceito relativo ao valor moral e ao sentido de eficácia e de competência de cada sujeito (Wells e Marwell, 1976), portanto, a auto-estima será consequência da relação entre os êxitos do sujeito e as suas pretensões, adquirindo valores positivos quando o sujeito se julga competente para atingir as suas pretensões/aspirações (Harter, 1985; James, 1892, cit. por Vaz Serra, 1988).

Mais ainda, de acordo com a definição de auto-estima de Rosenberg (1979), como sendo uma atitude que o sujeito apresenta relativamente ao self, esta pode ser positiva, quando o sujeito se avalia como tendo valor e sendo respeitado pelos outros, ou negativa, quando o sujeito se desvaloriza, se sente insatisfeito consigo próprio e não se respeita, nem se sente respeitado pelos outros.

Por sua vez, de uma forma sucinta, Dias e Fontaine (2001) apresentam a auto-estima como o "sentimento de se estar satisfeito consigo próprio e de se ter globalmente valor como pessoa" (p. 270), pelo que um indivíduo com elevada auto-estima tende a exibir como características uma elevada auto-aceitação, gostar de si próprio, auto-respeito e considerar-se uma pessoa de valor (Rosenberg, 1985).

Em suma, podemos concluir que o auto-conceito e a auto-estima se apresentam como constructos distintos mas interdependentes, uma vez que os vários estudos mostram que ambos se encontram relacionados positivamente e que o auto-conceito global não resulta da soma das suas diversas dimensões, sendo "uma síntese pessoal que tem a ver com o grau de satisfação em relação a si próprio" (Antunes, 1994, p. 73), síntese esta que pode ser identificada com a noção de auto-estima.

\section{Auto-conceito, auto-estima e sucesso académico}

Foi apenas nas décadas de oitenta e noventa que os factores motivacionais e afectivos se juntaram definitivamente aos cognitivos, para explicar, de forma mais completa, as situações de êxito-fracasso na aprendizagem escolar (González-Pienda, 2003). 
Fontaine (1990), no início da década de noventa, referiu-se à necessidade de recorrer a factores motivacionais para explicar as diferenças de rendimento, pois os factores cognitivos apenas conseguiam explicar $25 \%$ da variação dos resultados.

Assim, para um aluno obter bons resultados escolares precisaria de possuir capacidades para tal, mas também de ter vontade para atingir as suas metas (González-Pienda, 2003). Podemos, então, perceber a importância das variáveis motivacionais como sendo aquelas que dirigem a acção para a persistência, na prossecução de objectivos ou metas previamente fixadas pelo sujeito.

Mais concretamente, o interesse pelo estudo da relação entre o conceito de si, como variável motivacional, e os resultados escolares aumentou significativamente a partir da década de 70 , altura em que se começaram a atribuir os resultados escolares, o sucesso académico e a adaptação ao contexto escolar a factores para além da capacidade intelectual (Fontaine, 1991).

Contudo, os estudos que têm sido realizados para perceber o tipo de relação existente entre o auto-conceito e o rendimento escolar não são totalmente conclusivos: encontramos autores que apontam para a existência de uma relação recíproca (Cubero \& Moreno, 1992; Brown, Collins \& Schmidt, 1988; Helmke \& Van Aken, 1995; e Irwin, 1967, citados por Veiga, 1990; Marsh, 1988a; Marsh, 1990; Marsh \& Jackson, 1984; Marsh \& Yeung, 1987; Skaalvik \& Hagtvet, 1990) e outros que anunciam uma relação unilateral, isto é, defendem a existência de uma preponderância do rendimento sobre o auto-conceito ou vice-versa (Chapman \& Lambourne, 1990; Newman, 1984; citados por González-Pienda, 2003; Senos \& Diniz, 1998).

Por exemplo, Pereira (1991) advogou que o insucesso académico afecta o auto-conceito, mas apenas de forma sectorial, ou seja, segundo o autor, apesar de o aluno percepcionar insucesso em certas áreas, como por exemplo, a matemática, pode apresentar um bom auto-conceito geral. Este aspecto é explicado pelo autor por existir, no nosso país, "maior tolerância social, familiar ao insucesso" (p. 150). Por sua vez, num estudo com alunos do secundário, Haynes, Hamilton-Lee e Comer (1988) verificaram que os alunos com um rendimento escolar abaixo da média apresentavam níveis de auto-conceito significativamente inferiores aos de alunos com um rendimento acima da média.

Quanto à relação entre a auto-estima e o rendimento escolar, considera-se que os sentimentos, positivos ou negativos, que o indivíduo apresenta acerca de si próprio influenciam, significativamente, aspectos como o rendimento escolar, a saúde mental e o relacionamento com os outros (Jersild, 1952, cit. por Correia, 1991; Rosenberg, 1979). Autores como Baumeister e Tice (1985) consideraram que os sujeitos com baixa auto-estima têm tendência a transformar os resultados negativos em resultados pouco impor- 
tantes, porque passageiros. E, posteriormente, Baumeister, Tice e Hutton (1989) apresentaram os sujeitos com baixa auto-estima como sendo moderadamente persistentes na realização das tarefas, explicando este facto pela necessidade de remediar as dificuldades, evitando resultados humilhantes.

Já Senos e Diniz (1998), num estudo realizado com alunos do 9. ${ }^{\circ}$ ano de escolaridade, não encontraram diferenças significativas nos valores de auto-estima entre os alunos com melhores e piores resultados escolares. Em contrapartida, Peixoto (1998), num estudo realizado com sujeitos do distrito de Braga a frequentar o 9. ${ }^{\circ}$ ano de escolaridade, verificou que a auto-estima global e o sucesso escolar se encontravam positivamente correlacionados entre si $(r=0,45)$, embora não se possa falar de uma relação de causa e efeito, visto que outras variáveis poderão explicar a relação entre ambas. Neste estudo, o autor encontrou um número superior de reprovações nos sujeitos com baixa auto-estima e a percentagem de alunos com zero reprovações revelou-se superior nos alunos com elevada auto-estima.

Resumindo, parece ser mais evidente a existência de uma relação positiva entre o auto-conceito e o rendimento académico do que entre este e a auto-estima.

Auto-conceito e auto-estima na transição do ensino secundário para o superior

Os estudos sobre as mudanças que o auto-conceito e a auto-estima apresentam, ao longo do desenvolvimento dos indivíduos, mostram que existem períodos em que as mudanças são mais marcantes, períodos estes que parecem implicar alterações de contexto, que obrigam a transformações internas, fundamentais na avaliação das situações e na forma de encontrar meios para lhes fazer face. Assim, a transição do ensino secundário para o ensino superior assume especificidades que se podem traduzir em instabilidade no desenvolvimento, nomeadamente da auto-estima (Harter, 1983; Kohut, 1987, cit. por Dias \& Fontaine, 2001) e do auto-conceito.

Duarte e Faria (submetido a publicação), num estudo realizado com estudantes universitários, verificaram que o auto-conceito nas dimensões de pares do sexo oposto e global era menor nos alunos mais novos (a frequentar o $1 .^{\circ}$ ano) do que nos alunos mais velhos (do $3 .^{\circ}, 4 .^{\circ}$ e $5 .^{\circ}$ anos), o que mostra que, na fase de transição, os alunos se percebem como menos competentes para estabelecer relações com o sexo oposto, percepcionando-se, ainda, globalmente, como menos competentes. No entanto, o mesmo não se verificou na dimensão verbal e de assuntos escolares, em que os alunos do $1 .^{\circ}$ ano apresentaram valores significativamente superiores, resultado este que foi interpretado pelas autoras como indicador do maior investimento dos alunos dos últimos anos em aspectos do curso relacionados com a apli- 
cação das matérias aos contextos profíssionais, bem como com o ingresso no mundo do trabalho, em detrimento do investimento na aprendizagem de conteúdos académicos.

Quanto à relação que é possível estabelecer entre o auto-conceito e outras variáveis, Belo, Faria e Almeida (1998), num estudo realizado com estudantes do $1 .^{\circ}$ ano de várias licenciaturas da Universidade do Minho, verificaram que o auto-conceito académico, social e global se correlacionam positivamente com as dimensões de adaptação ao ensino superior (pessoal, relação com os outros e contexto académico): as subescalas de matemática, a relação com os pais e a língua materna foram as que apresentaram correlações mais baixas, enquanto o auto-conceito académico foi o que apresentou uma maior correlação com as várias dimensões de adaptação ao ensino superior.

Por fim, no que respeita à auto-estima, Dias e Fontaine (2001), num estudo realizado com estudantes universitários do $3 .^{\circ}$ ano, verificaram que a auto-estima é superior nos estudantes que estabelecem melhores relações com os seus pais, bem como nos jovens que estabelecem relações de namoro.

\section{Atribuições e dimensões causais: definição e delimitação conceptual}

A perspectiva atribucional centra o seu interesse na percepção do sujeito acerca das causas dos acontecimentos, sendo as atribuições causais determinantes dos comportamentos motivados, logo, dos comportamentos orientados para um determinado objectivo (Weiner, 1980). De acordo com Weiner (1980, 1985, 1986a, 1986b, 1990, 1992), um dos autores mais representativos da perspectiva atribucional, a necessidade de o sujeito compreender as suas acções, especialmente as que ocorrem pela primeira vez, impele-o a procurar as causas para esses acontecimentos. Esta procura das causas resulta da construção cognitiva por parte do sujeito, actor e observador da situação (Weiner, 1985), permitindo compreender os acontecimentos e, assim, prever o seu comportamento futuro (Fontaine, 1986).

Os primeiros estudos desenvolvidos para identificar as causas atribuídas por diferentes sujeitos a situações de sucesso e fracasso salientaram, como mais referidas, quatro tipos de causas: capacidade, esforço, sorte e dificuldade da tarefa (Weiner, Frieze, Kukla, Reed, Rest \& Rosenbaum, 1971). Posteriormente, embora uma infinidade de causas tenha sido documentada, as quatro causas referidas anteriormente têm continuado a predominar nos relatos dos sujeitos, especialmente as causas relativas à capacidade e ao esforço.

Weiner, atendendo à diversidade de atribuições causais identificadas e no sentido de as organizar e sistematizar, desenvolveu uma categorização 
com três dimensões causais bipolares diferentes (Faria, 1998b; Weiner, 1979, 1986a, 1986b, 1992, 1994), a saber: (i) o locus de causalidade (interno vs. externo), que corresponde à localização (interna ou externa) da causa percebida pelo actor (Weiner, 1986b); (ii) a estabilidade (estável vs. instável), que se refere à duração temporal da causa (Weiner, 1986b); e (iii) a controlabilidade (controlável vs. incontrolável), que corresponde à possibilidade de a causa vir a ser alterada pelo sujeito (Weiner, 1986b) e que surge no sentido de clarificar algumas dúvidas que permaneciam com a classificação bidimensional (locus e estabilidade), permitindo relacionar o locus e o controlo e, assim, categorizar causas que, apesar de internas, possam ser incontroláveis, como, por exemplo, a capacidade e o humor.

Refira-se, ainda, que a estas três dimensões foram acrescentadas mais duas - a intencionalidade e a globalidade -, que não serão aqui desenvolvidas, dado o menor impacto que têm revelado, a par da menor fundamentação empírica de que têm sido alvo (Brewin, 1985, cit. por Faria, 1998b; Fontaine, 1986).

\section{Atribuições, dimensões causais e sucesso académico}

No contexto educativo, parece interessante explorar a existência de relações entre as atribuições causais e o sucesso escolar, já que se tem verificado que os resultados de fracasso, inesperados ou atípicos, suscitam maior procura causal do que os sucessos ou os resultados esperados (Faria, 1998a). Da mesma forma, existem evidências que apontam no sentido de os alunos que apresentam resultados escolares baixos tenderem a procurar explicações causais que, simultaneamente, os ajudem a compreender o que aconteceu e a proteger a sua imagem pessoal e respectiva auto-estima.

Assim, podemos concluir que tem sido referida, por diversos autores, a existência de uma relação entre o rendimento escolar e as atribuições causais, muito embora persistam dúvidas acerca do tipo de relação, se directa ou indirecta, ou ainda se uni ou bilateral. Mais ainda, na análise dos diferentes estudos tem-se encontrado alguma variedade no tipo de causas consideradas para explicar os resultados, especialmente os de fracasso.

\section{Atribuições e dimensões causais na transição do ensino secundário para o superior}

O estudo das atribuições causais tem ocorrido, quer no nosso país, quer no estrangeiro, especialmente com alunos do ensino básico ou que se encontram no início do ensino secundário, pelo que são muito escassos os estudos sobre esta problemática realizados com alunos em transição do ensino secundário para o ensino superior ou em alunos do ensino superior. 
Por sua vez, os poucos estudos conhecidos sobre as atribuições causais realizados com alunos em transição do ensino secundário para o ensino superior apresentam resultados diversos, até porque alguns desses estudos se centram em aspectos muito específicos da aprendizagem, como veremos de seguida.

Assim, no nosso país existem alguns estudos sobre esta temática, realizados com alunos do $11 .^{\circ}$ ano de escolaridade (Faria, 1995, 1999), que permitiram concluir acerca da diferenciação das atribuições causais consoante o nível socioeconómico (NSE), salientando-se que os alunos de NSE alto recorrem, frequentemente, a atribuições internas para explicar os seus resultados académicos.

A nível do ensino superior, Relvas, Vaz Serra, Saraiva e Coelho (1984), numa investigação acerca da dimensão de locus de controlo, não encontraram diferenças significativas entre os sexos. Por sua vez, estes autores verificaram que, para explicar os diferentes comportamentos, os factores de controlo externo são percebidos como mais importantes pelos estudantes portugueses do que pelos estudantes americanos, apresentando estes maior tendência para evidenciar factores de controlo pessoal; ainda a nível dos factores externos, verificaram que os alunos portugueses valorizam mais a sorte e o destino, enquanto os alunos americanos valorizam mais o controlo atribuído a figuras de autoridade.

A este propósito, outros autores consideraram o papel das atribuições na sua relação com aspectos muito específicos da aprendizagem, como é o caso do estudo desenvolvido por Takahashi (2004), que realizou uma investigação com estudantes universitários do Japão, para averiguar que factores eram apontados pelos mesmos para explicar as suas dificuldades em aprender uma segunda língua, neste caso, o inglês, tendo verificado que existiam cinco factores principais, responsáveis pelas dificuldades na aprendizagem do inglês - por ordem de importância: o ambiente, a capacidade, a dificuldade da tarefa, o esforço e a orientação para a tarefa.

\section{Método}

\section{Amostra}

A amostra observada com o objectivo de estudar as relações entre as características motivacionais e o sucesso académico era constituída por 649 alunos do $12 .^{\circ}$ ano, sendo $60,4 \%$ do sexo feminino e $39,6 \%$ do sexo masculino, que frequentavam os diversos agrupamentos de estudos - Científico-Natural (54,5\%), Humanidades (19,4\%), Económico-Social (18,5\%) e Artes $(3,5 \%)-$, ou que frequentam Cursos Profissionais $(4,0 \%)$. A maior 
parte dos alunos optou pela Via Geral $(73,7 \%)$, enquanto $26,3 \%$ optaram pela Via Tecnológica ou Profissional. Em termos de estatuto social, 17,3\% eram alunos cujo agregado familiar apresentava um Nível Socioeconómico (NSE) alto, 41,1\% um NSE médio, 29,1\% um NSE médio-baixo e 12,0\% um NSE baixo.

Por sua vez, para estudar a mudança intra-individual nas características motivacionais, utilizou-se uma amostra de 62 alunos do $1 .^{\circ}$ ano do ensino superior, oriundos da amostra anterior, e que foram observados uma segunda vez, após a transição para o ensino superior. Destes, $60,6 \%$ eram do sexo feminino e $39,4 \%$ do sexo masculino; $59,1 \%$ frequentaram, no ensino secundário, o agrupamento Científico-Natural, 7,6\% Artes, 24,2\% Económico-Social e 9,1\% Humanidades; $87,9 \%$ frequentaram Cursos Gerais e 12,1\% Cursos Tecnológicos ou Profissionais. Quanto ao NSE do agregado familiar, $56,3 \%$ pertenciam ao nível baixo, $25,0 \%$ ao nível médio e $18,8 \%$ ao nível alto.

\section{Instrumentos}

Para a recolha dos dados, utilizaram-se os seguintes instrumentos de avaliação: (i) a adaptação portuguesa de Faria e Fontaine (1992) do Self-Description Questionnaire III (SDQ III), de Marsh e O'Niell (1984), com 136 itens que permitem a avaliação do auto-conceito académico, não académico, global e total, utilizando uma escala de resposta de tipo Likert, com 8 pontos, desde "Concordo Totalmente" até "Discordo Totalmente"; (ii) a Rosenberg Self-Esteem Scale (RSES), de Rosenberg (1965), com 10 itens que permitem avaliar a auto-estima global, utilizando uma escala de resposta de tipo Likert, com 8 pontos, desde "Concordo Totalmente" até "Discordo Totalmente"; (iii) o Questionário de Atribuições e Dimensões Causais (QADC), adaptado de Faria (1998) por Azevedo e Faria (2003), com 28 itens para cada uma das 4 dimensões - Influência, Locus, Estabilidade e Controlabilidade - e utilizando uma escala de resposta de tipo Likert, com 4 pontos, desde "Nenhuma Influência" até "Muita Influência", "Totalmente Externo" a "Totalmente Interno", "Totalmente Estável" a "Totalmente Instável" e "Não Controlo Totalmente" a "Controlo Totalmente", respectivamente para cada uma das referidas dimensões; (iv) os Questionários Sociodemográficos I e II (QSD I e II), construídos para este estudo, para recolher informação sobre a identificação, o agregado familiar, os antecedentes escolares e as expectativas dos participantes.

\section{Procedimento}

Os instrumentos foram administrados colectivamente aos alunos do $12 .^{\circ}$ ano de diversos grupos-turmas, durante o horário escolar normal, com 
transmissão oral das instruções. Para o estudo da mudança intra-individual, os alunos, após vivenciarem a transição, foram convidados a responder aos mesmos instrumentos, tendo sido sempre garantida a confidencialidade.

\section{Análise e discussão da relação entre características motivacio- nais e sucesso}

\section{Auto-conceito e sucesso académico}

Pela análise do Quadro 1, podemos observar que as correlações significativas entre as subescalas do auto-conceito (AC) e as notas obtidas nas três disciplinas aqui analisadas (Português, Matemática e Educação Física) são todas positivas e moderadas.

Por sua vez, melhores notas a Português estão associadas a percepções de maior competência nos domínios Matemático, Verbal, Assuntos Escolares em Geral e Académico, a percepções de maior Honestidade, a um melhor $A C$ Não Académico, a um melhor $A C$ Social e a um melhor $A C$ Total. Mais ainda, são os alunos que se consideram mais competentes a nível académico, não académico e social que obtêm melhores resultados a Português, pelo que o domínio da língua portuguesa parece depender da forma como o sujeito pensa acerca de si, quer a nível de resultados académicos, quer a nível não académico e social.

Já as melhores notas em Matemática estão associadas a um melhor Auto-conceito no domínio Matemático, Assuntos Escolares em Geral e Académico, a um melhor Auto-conceito de Honestidade e a um melhor Auto-conceito Total. Estes resultados mostram que os alunos que se percepcionam como mais competentes, especialmente a nível académico, apresentam melhores resultados a Matemática, aspecto este importante, pois os cursos que consideram a matemática como uma das disciplinas específicas permitem o prosseguimento de estudos num número superior de cursos superiores.

Quanto às notas em Educação Física, as melhores estão associadas a percepções de maior Competência Física, isto é, os alunos que se percepcionam como mais competentes a nível físico apresentam um melhor desempenho nessa disciplina.

Assim, globalmente, estes resultados apontam mais particularmente para uma relação entre os resultados académicos e o auto-conceito académico, confirmando os observados em investigações anteriores (Marsh, 1986, 1988b, 1989; Marsh, Byrne \& Shavelson, 1988), no entanto, tal tendência contraria alguns estudos realizados no nosso país (Veiga, 1989), pois 
nem sempre se observou uma correlação entre os resultados académicos e o auto-conceito académico.

Quadro 1-Correlações entre o auto-conceito, a auto-estima e as notas escolares

\begin{tabular}{|c|c|c|c|c|c|c|c|c|}
\hline & \multicolumn{2}{|c|}{$\begin{array}{c}\text { Nota a Português } \\
\text { no } 12 .^{\circ} \text { ano }\end{array}$} & \multicolumn{2}{|c|}{$\begin{array}{l}\text { Nota a Matemá- } \\
\text { tica no } 12 .^{\circ} \text { ano }\end{array}$} & \multicolumn{2}{|c|}{$\begin{array}{c}\text { Nota a } \\
\text { Ed. Física } \\
\text { no 12. }{ }^{\circ} \text { ano }\end{array}$} & \multicolumn{2}{|c|}{$\begin{array}{c}\text { Nota de } \\
\text { Candidatura }\end{array}$} \\
\hline & $r$ & $N$ & $r$ & $N$ & $r$ & $N$ & $r$ & $N$ \\
\hline Auto-conceito Matemática & $0,171 * *$ & 428 & $0,124 *$ & 270 & $-0,036$ & 450 & $-0,127$ & 230 \\
\hline Auto-conceito Verbal & $0,205^{* *}$ & 432 & 0,057 & 276 & 0,000 & 455 & 0,047 & 235 \\
\hline Ass. Escolares em Geral & $0,228 * *$ & 429 & $0,172 * *$ & 275 & $-0,020$ & 452 & $-0,043$ & 232 \\
\hline Resolução de Problemas & 0,082 & 432 & 0,072 & 273 & 0,011 & 454 & $-0,013$ & 235 \\
\hline Competência Física & $-0,046$ & 429 & $-0,015$ & 272 & $0,106 *$ & 452 & $-0,053$ & 233 \\
\hline Aparência Física & 0,028 & 425 & $-0,007$ & 266 & 0,016 & 447 & 0,062 & 232 \\
\hline Pares do mesmo sexo & 0,057 & 423 & 0,084 & 270 & 0,032 & 446 & $-0,014$ & 230 \\
\hline Pares do sexo oposto & 0,019 & 430 & 0,022 & 273 & 0,056 & 452 & 0,127 & 234 \\
\hline Pais & 0,040 & 433 & 0,051 & 275 & 0,000 & 456 & 0,010 & 234 \\
\hline Valores Espirituais/Religião & 0,073 & 430 & 0,029 & 272 & $-0,015$ & 451 & 0,028 & 235 \\
\hline Honestidade/Fiabilidade & $0,170 * *$ & 426 & $0,146 *$ & 273 & 0,034 & 448 & 0,079 & 231 \\
\hline Estabilidade Emocional & $-0,017$ & 429 & $-0,031$ & 274 & 0,031 & 452 & $-0,027$ & 234 \\
\hline Global & 0,045 & 428 & 0,019 & 272 & 0,028 & 451 & 0,090 & 236 \\
\hline Auto-conceito Académico & $0,249 * *$ & 420 & $0,151 *$ & 269 & $-0,027$ & 442 & $-0,072$ & 225 \\
\hline Auto-Conceito Não Académico & $0,148 * *$ & 392 & 0,091 & 250 & 0,060 & 411 & 0,042 & 215 \\
\hline Auto-Conceito Físico & $-0,012$ & 421 & $-0,012$ & 264 & 0,077 & 443 & 0,005 & 230 \\
\hline Auto-Conceito Social & $0,114 *$ & 418 & 0,110 & 266 & 0,055 & 440 & 0,071 & 227 \\
\hline Auto-Conceito Total & $0,300 * *$ & 383 & $0,133 *$ & 246 & 0,030 & 402 & 0,023 & 211 \\
\hline Auto-estima & 0,063 & 426 & 0,032 & 272 & 0,016 & 448 & 0,060 & 231 \\
\hline
\end{tabular}

Nota: ${ }^{*} p<0,05 ; * * p<0,01$

OBS.: algumas instituições não forneceram as notas escolares, alegando tratar-se de informação confidencial.

Por sua vez, o facto de termos encontrado uma correlação positiva entre dimensões específicas do auto-conceito, como o AC Matemática e o $A C$ Verbal, e o rendimento académico corrobora, igualmente, resultados observados anteriormente (Byrne, 1986; Byrne \& Shavelson, 1986; Marsh, 1984, 1990; Marsh, Parker \& Barnes, 1985). 
São ainda de salientar as associações que surgem entre as notas escolares e os domínios de competência que são afins a cada disciplina, de tal modo que a nota de Matemática está associada ao $A C$ Matemática, ao AC Assuntos Escolares em Geral e ao AC Académico; a nota de Português está associada ao AC Verbal, ao AC Assuntos Escolares em Geral e ao $A C$ Académico; e a nota de Educação Física está associada ao $A C$ Competência Física.

Por fim, refira-se que não se verificam correlações significativas entre as subescalas de auto-conceito e a nota de candidatura ao ensino superior.

\section{Auto-estima e sucesso académico}

As correlações das notas escolares com a Auto-estima Global não são significativas (Quadro 1), o que contraria os resultados de outras investigações que apontam para o facto de os sentimentos que o sujeito apresenta sobre si mesmo poderem influenciar o rendimento académico (Jersild, 1952, cit. por Correia, 1991; Peixoto, 1998; Rosenberg, 1979). Contudo, deve-se salientar que estes resultados mostram que não são tanto as percepções de competência global e de valor pessoal que são associadas pelos alunos aos resultados da sua realização escolar, mas sim as suas percepções de competência no domínio de realização directamente relacionado com a disciplina em questão.

\section{Atribuições e dimensões causais e sucesso académico}

Quando se correlacionam as dimensões causais com as notas escolares (Quadro 2), verificamos que apenas a correlação entre a dimensão causal de Estabilidade e a nota a Português no $12 .^{\circ}$ ano é significativa. Esta correlação, sendo moderada, é negativa, indicando que a melhores resultados nesta disciplina estão associadas percepções de maior estabilidade (a Estabilidade está cotada no sentido da maior instabilidade, logo, maior pontuação corresponde a maior instabilidade das causas). Quanto à ausência de correlações significativas entre as restantes dimensões e as notas, verifica-se que, para estes alunos, as percepções de maior ou menor Influência, Internalidade e Controlabilidade das causas não estão associadas aos respectivos resultados escolares.

Assim, salienta-se que, contrariamente ao observado noutros estudos (Faria, 1998b), a controlabilidade não mostrou ser a dimensão mais relevante, no que respeita aos resultados académicos: na verdade, neste estudo, o facto de o sujeito pensar que controla ou não os seus resultados não parece apresentar qualquer efeito nos resultados escolares obtidos. 
Quadro 2 - Correlações entre as dimensões causais e as notas escolares

\begin{tabular}{|c|c|c|c|c|c|c|c|c|}
\hline & \multicolumn{2}{|c|}{$\begin{array}{c}\text { Nota a Português } \\
\text { no 12. }{ }^{\circ} \text { ano }\end{array}$} & \multicolumn{2}{|c|}{$\begin{array}{c}\text { Nota a Matemática } \\
\text { no } 12 .^{\circ} \text { ano }\end{array}$} & \multicolumn{2}{|c|}{$\begin{array}{c}\text { Nota a Ed. Física } \\
\text { no 12. }\end{array}$} & \multicolumn{2}{|c|}{ Nota de Candidatura } \\
\hline & $r$ & $N$ & $r$ & $N$ & $r$ & $N$ & $r$ & $N$ \\
\hline Influência & 0,058 & 145 & 0,085 & 107 & 0,093 & 151 & 0,098 & 68 \\
\hline Locus & 0,107 & 145 & 0,146 & 109 & 0,094 & 151 & 0,049 & 69 \\
\hline Estabilidade & $-0,204 *$ & 146 & $-0,158$ & 108 & $-0,098$ & 152 & $-0,012$ & 69 \\
\hline Controlabilidade & $-0,075$ & 152 & 0,052 & 113 & $-0,109$ & 158 & $-0,102$ & 74 \\
\hline
\end{tabular}

\section{Análises de regressão linear}

Com o objectivo de clarificar o papel das variáveis motivacionais na explicação do sucesso escolar, foram realizadas análises de regressão linear, tomando como preditores as várias dimensões do auto-conceito, a auto-estima global e as dimensões causais: foram, então, testados vários modelos para estudar a influência destas variáveis nas notas de Português, de Matemática e de Educação Física do $12 .^{\circ}$ ano e, finalmente, na nota de candidatura ao ensino superior, de cujos resultados só apresentaremos os significativos, logo, apenas para a nota a Português e a Matemática.

Assim, a análise de regressão para o modelo explicativo da nota de Português revela que o resultado é significativo, explicando cerca de $35 \%$ da variabilidade da nota nesta disciplina: as dimensões $A C$ Matemática, $A C$ Verbal e AC Assuntos Escolares em Geral são as variáveis preditoras que mostram influenciar positivamente a nota a Português, sendo que o $A C$ Verbal é a subescala que tem o maior poder preditivo (Quadro 3).

Por sua vez, o modelo analisado para a nota de Matemática também revela um resultado significativo, explicando cerca de $10 \%$ da variabilidade desta nota: a dimensão de AC Assuntos Escolares em Geral é a única com influência positiva na nota de Matemática.

Deste modo, verifica-se que a maioria dos resultados das análises de regressão vai no sentido dos resultados encontrados nos estudos correlacionais entre o auto-conceito/auto-estima e as notas escolares.

Em suma, encontramos uma grande capacidade explicativa por parte das subescalas de auto-conceito ligadas ao contexto escolar (que podemos designar por $A C$ Académico), na disciplina de Português e na disciplina de Matemática, sendo o AC Assuntos Escolares em Geral a única variável com poder explicativo das referidas notas: assim, podemos afirmar que o auto- 
-conceito académico parece influenciar positivamente os resultados da realização nestas duas importantes disciplinas do currículo escolar.

Quadro 3 - Análises de regressão linear para a nota a Português (P) e a Matemática (M) no $12 .^{\circ}$ ano, tendo como variáveis preditoras o auto-conceito e a auto-estima $(\mathrm{N}=378)$

\begin{tabular}{lcccrrc}
\hline Preditores & $\boldsymbol{B e t a}_{\boldsymbol{P}}$ & $\boldsymbol{t}_{\boldsymbol{P}}$ & $\boldsymbol{p}_{\boldsymbol{P}}$ & $\boldsymbol{B e t a}_{\boldsymbol{M}}$ & $\boldsymbol{t}_{\boldsymbol{M}}$ & $\boldsymbol{p}_{\boldsymbol{M}}$ \\
\hline Auto-Conceito de Matemática & $0,244^{* * *}$ & 4,544 & $0,000^{*}$ & 0,101 & 1,255 & 0,211 \\
Auto-Conceito Verbal & $0,270^{* * *}$ & 4,725 & $0,000^{*}$ & $-0,113$ & $-1,336$ & 0,183 \\
Assuntos Escolares/Académ. & $0,241^{* * *}$ & 3,603 & $0,000^{*}$ & $0,256^{*}$ & 2,439 & $0,015^{*}$ \\
em Geral & $-0,053$ & $-0,877$ & 0,381 & 0,053 & 0,578 & 0,564 \\
Resolução de Problemas & $-0,130^{* *}$ & $-2,750$ & 0,006 & $-0,033$ & $-0,452$ & 0,652 \\
Competência Física & $-0,022$ & $-0,377$ & 0,706 & $-0,082$ & $-1,008$ & 0,314 \\
Aparência Física & $-0,001$ & $-0,026$ & 0,980 & 0,122 & 1,549 & 0,123 \\
Pares do Mesmo Sexo & $-0,062$ & $-1,192$ & 0,234 & 0,031 & 0,398 & 0,691 \\
Pares do Sexo Oposto & 0,049 & 0,958 & 0,339 & 0,043 & 0,520 & 0,604 \\
Pais & 0,063 & 1,344 & 0,180 & $-0,066$ & $-0,947$ & 0,345 \\
Valores Espirituais/Religião & 0,052 & 1,043 & 0,298 & 0,044 & 0,573 & 0,567 \\
Honestidade/Fiabilidade & $-0,063$ & $-1,106$ & 0,269 & $-0,072$ & $-0,847$ & 0,398 \\
Estabilidade Emocional & $-0,164$ & $-1,879$ & 0,061 & $-0,089$ & $-0,720$ & 0,472 \\
Global & 0,119 & 1,374 & 0,170 & $-0,033$ & $-0,255$ & 0,799 \\
Auto-Estima & & & & &
\end{tabular}

Nota: Modelo Português: $R^{2}=0,349 ; F=13,877 ; g l=14 ; p=0,000 ; \mathrm{N}=378$; Modelo Matemática: $R^{2}=0,104 ; F=1,882 ; g l=14 ; p=0,029 ; \mathrm{N}=243$.

Legenda: $\operatorname{Beta}_{P}$ (Beta obtido para a disciplina de Português); $t_{P}$ (t obtido para a disciplina de Português); $p_{p}$ (p obtido para a disciplina de Português); Beta ${ }_{M}$ (Beta obtido para a disciplina de Matemática); $t_{M}\left(\mathrm{t}\right.$ obtido para a disciplina de Matemática); $p_{M}$ (p obtido para a disciplina de Matemática).

Já no que se refere à auto-estima, verifica-se que esta não contribui, por si só, para explicar nenhuma das notas.

Foram, ainda, realizadas análises de regressão linear, assumindo como variáveis preditoras o nível de influência das causas atribuídas aos resultados escolares e as três dimensões causais (Locus de Causalidade, Estabilidade e Controlabilidade), mas também não se verificou nenhum resultado significativo.

\section{Análise e discussão dos resultados do estudo da mudança intra- -individual}

\section{Auto-conceito}

Como se pode verificar no Quadro 4, os alunos, após a entrada para o ensino superior, percepcionam-se globalmente de uma forma mais positiva, bem como em termos verbais, físicos e sociais (especialmente a nível do 
relacionamento que estabelecem com os pais): ou seja, além do desenvolvimento do auto-conceito geral, são potenciadas dimensões como a competência verbal (o que pode estar associado a um aumento da autonomia na aprendizagem), a aparência física (o que pode estar associado ao facto de os alunos possuírem mais tempo para investir na sua imagem física) e a relação com os pais (a entrada no ensino superior pode promover uma relação com os pais mais equilibrada, dado que coincide com uma fase do afastamento do grupo de amigos estabelecido anteriormente e com o início do estabelecimento de novas amizades, permanecendo os pais como um recurso constante).

A este propósito, Duarte e Faria (submetido a publicação), num estudo levado a cabo com alunos do ensino superior, verificaram que, a nível do Auto-conceito Global, os alunos do $1 .^{\circ}$ ano se percepcionavam como menos competentes do que os do $3 .^{\circ}, 4 .^{\circ}$ e $5 .^{\circ}$ anos, o que mostra a tendência para o auto-conceito aumentar durante a frequência do ensino superior: assim, o facto de a mudança de contexto potenciar uma mudança no auto-conceito pode significar que este constructo, apesar de manter uma certa constância, é vulnerável às mudanças contextuais.

\section{Auto-estima}

Relativamente à auto-estima, não foram encontradas diferenças significativas entre os resultados obtidos no $1 .^{\circ}$ momento e os obtidos no $2 .^{\circ}$ momento de avaliação (Quadro 4), o que corrobora resultados de estudos anteriores, que mostraram não haver variação da auto-estima em jovens adultos, em função da idade (Dias, 1996; Dias \& Fontaine, 2001). Ou seja, estes resultados parecem comprovar que a auto-estima se assume como um constructo mais estável do que o auto-conceito, sendo menos vulnerável às umdanças contextuais.

\section{Atribuições e dimensões causais}

Pela análise do Quadro 5, podemos verificar que entre o $1 .^{\circ}$ e o 2. ${ }^{\circ}$ momentos de avaliação (antes e após o ingresso no ensino superior), isto é, na transição do ensino secundário para o ensino superior, há um aumento na influência percepcionada das causas (Influência), bem como nas percepções da sua controlabilidade (Controlabilidade). Observamos ainda um decréscimo na Estabilidade, o que sugere que os alunos desenvolveram percepções de maior estabilidade das causas (como já referimos, a Estabilidade está cotada no sentido da maior instabilidade, ou seja, maior pontuação corresponde a maior instabilidade das causas). 
Quadro 4 - MANOVAS para medidas repetidas para o auto-conceito e a auto-estima, considerando como factor de diferenciação o tempo (transição do ensino secundário para o ensino superior)

\begin{tabular}{|c|c|c|c|c|c|c|c|c|c|c|}
\hline & \multirow{2}{*}{$N$} & \multirow{2}{*}{$\begin{array}{l}\text { Soma dos } \\
\text { Quadrados }\end{array}$} & \multirow{2}{*}{$\begin{array}{c}\text { Média } \\
\text { Quadrática }\end{array}$} & \multirow{2}{*}{$g l$} & \multirow{2}{*}{$F$} & \multirow{2}{*}{$p$} & \multicolumn{2}{|c|}{$1 .^{\circ}$ momento } & \multicolumn{2}{|c|}{$2 .^{\circ}$ momento } \\
\hline & & & & & & & $M$ & $D P$ & $M$ & $D P$ \\
\hline $\begin{array}{l}\text { AC } \\
\text { Matemática }\end{array}$ & 62 & 20,976 & 20,976 & 1 & 0,564 & 0,455 & 51,9 & 14,9 & 51,0 & 15,5 \\
\hline AC Verbal & 62 & 355,645 & 355,645 & 1 & 9,749 & $0,003^{*}$ & 55,4 & 10,7 & 58,8 & 9,7 \\
\hline $\begin{array}{l}\text { Assuntos } \\
\text { Escolares }\end{array}$ & 63 & 34,571 & 34,571 & 1 & 0,679 & 0,413 & 56,4 & 10,3 & 57,4 & 9,6 \\
\hline $\begin{array}{l}\text { Resol. de } \\
\text { Problemas }\end{array}$ & 62 & 20,161 & 20,161 & 1 & 0,995 & 0,322 & 53,2 & 8,5 & 54,0 & 9,0 \\
\hline $\begin{array}{l}\text { Competência } \\
\text { Física }\end{array}$ & 62 & 0,202 & 0,202 & 1 & 0,008 & 0,930 & 59,3 & 14,5 & 59,3 & 14,8 \\
\hline $\begin{array}{l}\text { Aparência } \\
\text { Física }\end{array}$ & 61 & 321,344 & 321,344 & 1 & 8,628 & $0,005 *$ & 49,6 & 12,9 & 52,9 & 12,7 \\
\hline $\begin{array}{l}\text { Pares } \\
\text { Mesmo Sexo }\end{array}$ & 63 & 31,500 & 31,500 & 1 & 0,621 & 0,434 & 62,0 & 9,3 & 61,0 & 10,3 \\
\hline $\begin{array}{l}\text { Pares Sexo } \\
\text { Oposto }\end{array}$ & 61 & 30,500 & 30,500 & 1 & 0,674 & 0,415 & 60,2 & 11,2 & 61,2 & 9,9 \\
\hline Pais & 61 & 283,574 & 283,574 & 1 & 7,307 & $0,009^{*}$ & 58,8 & 12,5 & 61,8 & 11,5 \\
\hline $\begin{array}{l}\text { Valores } \\
\text { Espirituais }\end{array}$ & 61 & 32,533 & 32,533 & 1 & 0,445 & 0,507 & 62,8 & 16,5 & 63,8 & 19,5 \\
\hline Honestidade & 62 & 0,290 & 0,290 & 1 & 0,010 & 0,921 & 80,6 & 6,6 & 80,7 & 7,7 \\
\hline $\begin{array}{l}\text { Estabil. } \\
\text { Emocional }\end{array}$ & 62 & 15,613 & 15,613 & 1 & 0,352 & 0,555 & 44,3 & 11,4 & 45,0 & 12,3 \\
\hline $\begin{array}{l}\text { Global ou } \\
\text { Geral }\end{array}$ & 62 & 419,226 & 419,226 & 1 & 4,198 & $0,045^{*}$ & 71,1 & 15,4 & 74,8 & 14,2 \\
\hline $\begin{array}{l}\text { AC } \\
\text { Académico }\end{array}$ & 61 & 476,074 & 476,074 & 1 & 2,903 & 0,094 & 163,5 & 28,1 & 167,5 & 26,3 \\
\hline $\begin{array}{l}\text { AC Não } \\
\text { Académico }\end{array}$ & 54 & 1571,704 & 1571,704 & 1 & 1,790 & 0,187 & 533,6 & 51,0 & 541,2 & 60,5 \\
\hline AC Físico & 60 & 264,033 & 264,033 & 1 & 3,329 & 0,073 & 108,7 & 23,2 & 111,6 & 22,9 \\
\hline AC Social & 60 & 197,633 & 197,633 & 1 & 1,005 & 0,320 & 181,5 & 24,6 & 184,1 & 23,6 \\
\hline AC Total & 53 & 6704,236 & 6704,236 & 1 & 3,247 & 0,077 & 771,3 & 80,9 & 787,2 & 85,7 \\
\hline Auto-estima & 58 & 96,862 & 96,862 & 1 & 1,377 & 0,245 & 59,7 & 12,2 & 61,5 & 10,1 \\
\hline
\end{tabular}

Em suma, podemos dizer que a transição para o ensino superior, representando uma importante mudança de contexto de ensino, parece promover melhores percepções de si próprio em vários domínios, bem como 
uma maior consciência da influência das causas e da sua estabilidade e controlabilidade.

Quadro 5-MANOVAS para medidas repetidas para as dimensões causais, considerando como factor de diferenciação o tempo (transição do ensino secundário para o ensino superior)

\begin{tabular}{|c|c|c|c|c|c|c|c|c|c|c|}
\hline & \multirow{2}{*}{$N$} & \multirow{2}{*}{$\begin{array}{l}\text { Soma dos } \\
\text { Quadrados }\end{array}$} & \multirow{2}{*}{$\begin{array}{c}\text { Média } \\
\text { Quadrática }\end{array}$} & \multirow{2}{*}{$g l$} & \multirow[t]{2}{*}{$F$} & \multirow{2}{*}{$p$} & \multicolumn{2}{|c|}{$\begin{array}{c}1 .^{\circ} \\
\text { momento }\end{array}$} & \multicolumn{2}{|c|}{$\begin{array}{c}2 .^{\circ} \\
\text { momento }\end{array}$} \\
\hline & & & & & & & $M$ & $D P$ & $M$ & $D P$ \\
\hline Influência & 63 & 301,786 & 301,786 & 1 & 33,791 & 0,000 * & 41,3 & 3,7 & 44,4 & 5,3 \\
\hline Locus & 65 & 7,392 & 7,392 & 1 & 1,833 & 0,181 & 39,8 & 3,7 & 39,3 & 3,6 \\
\hline Estabilidade & 66 & 50,939 & 50,939 & 1 & 8,235 & $0,006^{*}$ & 35,1 & 3,9 & 33,9 & 4,0 \\
\hline Controlabilidade & 64 & 134,070 & 134,070 & 1 & 23,831 & $0,000 *$ & 36,8 & 3,8 & 38,9 & 3,1 \\
\hline
\end{tabular}

Nota: ${ }^{*} p<0,05$

\section{Conclusão}

Neste trabalho, da observação da relação entre as características motivacionais e o sucesso académico, podemos concluir que o auto-conceito é a variável motivacional que mais se correlaciona com o sucesso académico, pois encontramos uma correlação positiva e significativa entre algumas dimensões do auto-conceito académico e não-académico e as notas a Português e a Matemática.

Por sua vez, a nível das atribuições e dimensões causais, verificamos que apenas a estabilidade das causas se apresenta correlacionada significativa e negativamente com a nota à disciplina de Português.

Quanto ao facto de as notas às diferentes disciplinas poderem ser explicadas pelas características motivacionais, podemos concluir que $35 \%$ da variação da nota a Português e $10 \%$ da variação da nota a Matemática são explicadas pelo auto-conceito.

No que respeita ao estudo da mudança intra-individual das variáveis motivacionais, constata-se que, entre o $1 .^{\circ}$ e o $2 .^{\circ}$ momentos de avaliação, houve um aumento significativo em algumas dimensões do Auto-conceito (AC Verbal, AC Aparência Física, AC Relação com os Pais e AC Global), o mesmo não acontecendo com a Auto-estima, que, apesar de ter aumentado, a respectiva diferença não se revelou significativa. Já nas dimensões atribucionais de Influência e Controlabilidade foram encontradas diferenças significativas entre os dois momentos de avaliação. Verificou-se, ainda, um decréscimo na Estabilidade entre os dois referidos momentos de avaliação.

Finalmente, salientamos que o auto-conceito se relaciona com o sucesso académico, pelo que parece assumir-se como a variável motivacio- 
nal mais importante para potenciar a adaptação ao ensino superior: mas ainda, se é certo que a transição parece promover o auto-conceito em algumas das suas dimensões, não deixamos de considerar que o apoio aos alunos do $12 .^{\circ}$ ano, no sentido de promover a sua adaptação e sucesso académico no ensino superior, deve contemplar mais particularmente o auto-conceito a nível das dimensões sociais.

Quanto a futuras investigações, salientamos a necessidade de estudar o processo de transição do ponto de vista de outros intervenientes, nomeadamente dos professores e dos pais que lidam com os alunos nesta fase tão importante.

Finalmente, propomos que a transição, enquanto um processo dinâmico, seja alvo de um estudo contínuo e aprofundado, pois as mudanças tecnológicas do terceiro milénio, que impõem alterações na estrutura do ensino superior, e a diminuição demográfica de jovens no nosso país não deixarão de implicar, a curto prazo, novas questões psicossociais quanto à transição do ensino secundário para o ensino superior.

\section{Referências}

Antunes, C. (1994). O apoio social e o conceito de si próprio na adolescência. Tese de Mestrado. Faculdade de Psicologia e de Ciências da Educação da Universidade do Porto. Porto: Edição do autor.

Azevedo, A., \& Faria, L. (2001). Impacto das condições pessoais e contextuais na transição do ensino secundário para o ensino superior. Revista da UFP, 6, 257-269 .

Azevedo, A., \& Faria, L. (2003). Questionário de atribuições e dimensões causais: qualidades psicométricas no contexto escolar português. Revista Galego-Portuguesa de Psicoloxía e Education, 8 (Vol. 10), Ano 7, 1138-1663.

Baumeister, R. F., \& Tice, D. M. (1985). Self-esteem and responses to success and failure: Subsequent performance and intrinsic motivation. Journal of Personality, 53, 450-467.

Baumeister, R. F., Tice, D. M., \& Hutton, D. G. (1989). Self-representation, motivation and personality differences in self-esteem. Journal of Personality, 57, 547-579.

Belo, S., Faria, L., \& Almeida, L. (1998). Adaptação ao ensino superior: importância do auto-conceito dos estudantes. In L. Almeida, R. Gomes, P. Albuquerque, \& S. Caires (Eds.), Actas do IV Congresso Galaico-Português de Psicopedagogia (pp. 60-68). Braga: Universidade do Minho.

Bronfenbrenner, U. (1979). The ecology of human development. Cambridge: Harvard University Press.

Bronfenbrenner, U. (1981). L'ecologie expérimentale de l'éducation. In Beaudot (Org.), Sociologie de l'école - pour une analyse de l'établissement Scolaire. Paris: Bordas. 
Brown, J. D., Collins, R. L., \& Schmidt, G. W. (1988). Self-esteem and direct versus indirect forms of self-enhancement. Journal of Personality and Social Psychology, 55, 445-453.

Burns, R. B. (1982). Self-concept development and education. London: Dorset Press.

Byrne, B. M. (1986). Self-concept/academic achievement relations: An investigation of dimensionality, stability and causality. Canadian Journal of Behavioral Science, 18, 173-186.

Byrne, B. M., \& Shavelson, R. (1986). On the structure of adolescent self-concept. Journal of Educational Psychology, 78, 474-481.

Correia, M. L. (1991). Auto-percepção e auto-estima em crianças com competência no $1 .^{\circ}$ ciclo do ensino obrigatório. Psicologia, VIII (1), 45-55.

Cubero, R., \& Moreno, M. C. (1992). Relaciones sociales: família, escuela, compañeros, años escolares. In J. Palácios, A. Marclose \& C. Coll (Eds.), Desarrollo psicológico y educación, I - Psicologia evolutiva (4.a ed., pp. 285-296). Madrid: Alianza Editorial.

Dias, M. G. F. (1996). Tarefas desenvolvimentais e bem-estar de jovens. Tese de Doutoramento. Faculdade de Psicologia e Ciências da Educação da Universidade do Porto. Porto: Edição do autor.

Dias, M. G. F., \& Fontaine, A. M. (2001). Tarefas desenvolvimentais e bem-estar de jovens universitários (caps. 4, 6 e 7). Lisboa: Fundação Calouste Gulbenkian.

Duarte, A. M., \& Faria, L. (submetido a publicação). O auto-conceito em estudantes universitários de Desporto e Educação Física da Universidade do Porto.

Faria, L. (1995). Desenvolvimento diferencial das concepções pessoais de inteligência durante a adolescência. Dissertação apresentada para provas de doutoramento em Psicologia na Faculdade de Psicologia e de Ciências da Educação da Universidade do Porto. Porto: Edição do autor.

Faria, L. (1998a). Concepções pessoais de inteligência, atribuições e rendimento escolar. Psicologia, XII (1), 101-113.

Faria, L. (1998b). Desenvolvimento diferencial das concepções pessoais de inteligência durante a adolescência. Lisboa: Fundação Calouste Gulbenkian e Junta Nacional de Investigação Científica e Tecnológica.

Faria, L. (1999). Contextos sociais de desenvolvimento das atribuições causais: O papel do nível sócio-económico e da raça. Análise Psicológica, 2 (XVII), $265-$ -273 .

Faria, L., \& Fontaine, A. M. (1990). Avaliação do conceito de si próprio de adolescentes: Adaptação do SDQ I de Marsh à população portuguesa. Cadernos de Consulta Psicológica, 6, 97-106.

Faria, L., \& Fontaine, A. M. (1992). Estudo de adaptação do Self-Description Questionnaire III (SDQ III) a estudantes universitários portugueses. Psychologica, 8, 41-49.

Fleming, J. S., \& Courtner, B. E. (1984). The dimensionality of self-esteem: Hierarchical facet model revised measurement scales. Journal of Personality and Social Psychology, 46, 404-421. 
Fontaine, A. M. (1986). Motivation pour la réussite scolaire. Dissertação apresentada para provas de doutoramento em Psicologia na Faculdade de Psicologia e de Ciências da Educação da Universidade do Porto. Porto: Edição do autor.

Fontaine, A. M. (1990). Motivação e realização escolar. In B. P. Campos (Coord.), Psicologia do desenvolvimento e educação de jovens (pp. 93-132). Lisboa: Universidade Aberta.

Fontaine, A. M. (1991). Desenvolvimento do conceito de si próprio e realização escolar na adolescência. Psychologica, 5, 13-31.

González-Pienda, J. A. (2003). El rendimento escolar: uma análise de las variables que lo condicionan. Revista Galego-Portuguesa de Psicoloxía e Educación, 7 (9), 247-258.

Gurney, P. W. (1986). Self-esteem in the classroom: Theoretical perspectives and assessment issues. School Psychology International, 7, 199-209.

Harter, S. (1983). Developmental perspectives on the self-system. In E. M. Hetherington (Ed.), Handbook of child psychology: Socialization, personality, and social development (pp. 275-383). New York: Wiley.

Harter, S. (1985). Manual for the Self-Perception Profile for Children. (Revision of Perceived Competence Scale for Children). Denver: University of Denver.

Haynes, N. M., Hamilton-Lee, M., \& Comer, J. P. (1988). Differences in self-concept among high, average and low achieving high-school sophoromes. Journal of Social Psychology, 128, 259-260.

Marsh, H. W. (1984). Relations among dimensions of self-attribution, dimensions of self-concept and academic achievement. Journal of Educational Psychology, 76, 1291-1308.

Marsh, H. W. (1986). Global self-esteem: Its relation to specific facets of self-concept and their importance. Journal of Personality and Social Psychology, $51,1224-1236$.

Marsh, H. W. (1988a). Causal effects of academic self-concept on academic achievement: A reanalysis of Newman (1984). Journal of Experimental Education, 56, 100-104.

Marsh, H. W. (1988b). The content specificity of math and English anxieties: The high school and beyond study. Anxiety Research, 1, 137-149.

Marsh, H. W. (1989). Sex differences in the development of verbal and mathematics constructs: The high school and beyond study. American Educational Research Journal, 26, 191-225.

Marsh, H. W. (1990). Causal ordering of academic self-concept and academic achievement: A multi-wave, longitudinal panel analysis. Journal of Educational Psychology, 82 (4), 646-656.

Marsh, H. W., Byrne, B. M., \& Shavelson, R. J. (1988). A multifaceted academic self-concept: its hierarchical structure and its relation to academic achievement. Journal of Educational Psychology, 80, 366-380.

Marsh, H. W., \& Jackson, S. Q. (1984). Multidimensional self-concepts, masculinity and femininity as a function of women's involvement in athletics. Sydney: University of Sydney. 
Marsh, H. W., \& O’Niell, R. (1984). Self-Description Questionnaire III (SDQ III): The construct validity of multidimensional self-concept ratings by late-adolescents. Journal of Educational Measurement, 21, 153-174.

Marsh, H. W., Parker, J., \& Barnes, J. (1985). Multidimensional adolescent self-concepts: Their relationship to age, sex and academic measures. American Educational Research Council, 22, 422-444.

Marsh, H. W., \& Yeung, A. S. (1987). Causal effects of self-concept on academic achievement: structural equation models of longitudinal data. Journal of Educational Psychology, 89 (1), 41-54.

Peixoto, F. (1998). Auto-conceito (s), auto-estima e resultados escolares: a influência da repetência no(s) auto-conceito(s) e na auto-estima de adolescentes. In M. A. Martins (Ed.), Actas do IX Colóquio de Psicologia e Educação (pp. 51-69). Lisboa: ISPA.

Peixoto, F., \& Almeida, L. S. (1999). Escala de auto-conceito e auto-estima. In A. P. Soares, S. Araújo \& S. Caires (Orgs.), Avaliação Psicológica: Formas e Contextos. (Vol. VI, pp. 633-640). Braga: APPORT.

Pereira, F. (1991). Auto-conceito e resultados escolares na adolescência. Análise Psicológica, 2 (IX), 145-150.

Relvas, J., Vaz Serra, A., Saraiva, C., \& Coelho, I. (1984). Resultados da aplicação da Escala IPC de Levenson a estudantes universitários. Psiquiatria Clínica, 5 (3), 119-124.

Rosenberg, M. (1965). Society and the adolescent self-image. Princeton: Princeton University Press.

Rosenberg, M. (1979). Conceiving the self. Florida: Krieger Publishing Company.

Rosenberg, M. (1985). Self-concept and psychological well-being in adolescence. In R. L. Leahy (Ed.), The development of the self. New York: Academic Press.

Senos, J., \& Diniz, T. (1998). Auto-estima, resultados escolares e indisciplina. Estudo exploratório numa amostra de adolescentes. Análise Psicológica, 2, 267-276.

Shavelson, R. J., Hubner, J. J., \& Stanton, J. C. (1976). Self-concept: validation of construct interpretations. Review of Educational Research, 46, 407-441.

Skaalvik, E. M., \& Hagtvet, K. A. (1990). Academic achievement and self-concept: an analysis of causal predominance in a developmental perspective. Journal of Personality and Social Psychology, 58, 292-307.

Takahashi, S. (2004). The role of "attribution for success and failure" in second language reading by Japanese university students. Humanities and Social Sciences, 64 (7-A), 24-28.

Vaz Serra, A. (1986). O inventário clínico de Auto-Conceito. Psiquiatria Clínica, 7 (2), 67-84

Vaz Serra, A. (1988). Atribuição e auto-conceito. Psychologica, 1, 127-141.

Vaz Serra, A. (1999). O stress na vida de todos os dias. Coimbra: Gráfica de Coimbra Lda.

Vaz Serra, A., Firmino, H., \& Ramalheira, C. (1988). Estratégias de coping e auto-conceito. Psiquiatria Clínica, 9 (4), 317-322. 
Veiga, F. H. (1989). Escala de autoconceito: Adaptação portuguesa do "Piers-Harris Children's Self-Concept scale". Psicologia, VII (3), 275-284.

Veiga, F. H. (1990). Autoconceito e disrupção escolar dos jovens: Conceptualização, avaliação e diferenciação. Tese de Doutoramento. Universidade de Lisboa. Departamento de Educação da Faculdade de Ciências. Lisboa: Edição de autor.

Weiner, B. (1979). A theory of motivation for some classroom experiences. Journal of Educational Psychology, 71, 3-25.

Weiner, B. (1980). A cognitive (attribution)-emotion-action model of motivated behavior: An analysis of judgment of help-giving. Journal of Personality and Social Psychology, 39, 186-200.

Weiner, B. (1985). An attributional theory of achievement motivation and emotion. Psychological Review, 92, 548-573.

Weiner, B. (1986a). An attributional theory of achievement motivation and emotion. New York: Springer-Verlag.

Weiner, B. (1986b). Attribution, emotion, and action. In R. M. Sorrentino \& E. T. Higgins (Eds.), Handbook of motivation and cognition (pp. 281-312). New York: The Guilford Press.

Weiner, B. (1990). History of motivational research in education. Journal of Educational Psychology, 82, 616-622.

Weiner, B. (1992). Human motivation. Metaphors, theories and research. London: SAGE.

Weiner, B. (1994). Integrating social and personal theories of achievement striving. Review of Educational Research, 64, 557-573.

Weiner, B., Frieze, I. H., Kukla, A., Reed, L., Rest, S., \& Rosenbaum, R. M. (1971). Perceiving the causes of success and failure. Morristown, N.J.: General Learning Press.

Wells, E. L., \& Marwell, G. (1976). Self-esteem its conceptualisation and measurement. London: Sage Publications.

Wylie, R. C. (1974). The self-concept: A review of methodological considerations and measuring instruments (2. ${ }^{\mathrm{a}}$ Ed.). Lincoln: University of Nebraska Press. 